\title{
Cohort Study
}

\section{e Evaluation of Opioid Prescribing Habits Based on Analysis of a State Prescription Drug Monitoring Program}

John C. Alexander, MD', Julia Silge, $\mathrm{PhD}^{2}$, Stephanie Jones, MD', and Girish P. Joshi, MD¹

From: ${ }^{1}$ Department of Anesthesiology and Pain Management, University of Texas Southwestern, Dallas, TX; ${ }^{2}$ Stack Overflow, New York, NY

Address Correspondence: John C. Alexander, MD Department of Anesthesiology and Pain Management University of Texas Southwestern 5323 Harry Hines Blvd. Dallas, TX 75390-9068

E-mail: John.Alexander@ utsouthwestern.edu

Disclaimer: There was no external funding in the preparation of this manuscript. Conflict of interest: Each author certifies that he or she, or a member of his or her immediate

family, has no commercial association (i.e., consultancies, stock ownership, equity interest, patent/licensing arrangements, etc.) that might pose a conflict of interest in connection with the submitted manuscript.

Manuscript received: 11-14-2018 Revised manuscript received: 02-05-2019

Accepted for publication: 03-26-2019

Free full manuscript: www.painphysicianjournal.com
Background: The current opioid epidemic is perhaps the greatest public health crisis in the United States. Although multiple factors led to the rise of this epidemic, it is without question associated with the rise in opioid prescribing.

Objectives: Better understanding of the opioid prescribing may provide insights into populationlevel trends contributing to this epidemic, and opportunities to decrease the magnitude of opioid overdose-related death. Therefore we assessed trends in opioid prescribing habits based on analysis of the Texas Prescription Drug Monitoring Program (PDMP) and geographic, ethnic, and incomerelated data from the US Census Bureau.

Study Design: Multiple linear regression analysis of Texas PDMP and US Census Bureau data were performed to assess for correlations to opioid prescribing based on geographic, ethnic, income, and time-related variables.

Setting: All controlled substances prescribed in the state of Texas from April 2015 to May 2018 were analyzed.

Methods: We obtained data from the Texas PDMP for all controlled substances from April 2015 to May 2018. We performed multiple linear regression analysis of these data along with US Census Bureau data to assess for correlations based on geographic, ethnic, income, and time-related variables. We hypothesized that there would be substantial variability in opioid prescribing habits based on geographic, ethnic, and economic variables.

Results: Approximately 200 million pills of controlled substances were prescribed per month over the studied time frame. Overall, high geographic variability was noted, and this strongly correlated to race and ethnicity. Opioid prescribing increased along with the proportion of white residents within a county, but a similar negative correlation was noted with increasing Hispanic population proportion. This correlation was noted throughout the study period, but up until 2017, lower income levels among higher white population had even higher correlation with increased opioid prescribing. Cumulative opioid prescriptions throughout the state fell beginning in 2017.

Limitations: This analysis does not include opioids obtained illicitly or from prescriptions outside the state of Texas. The specificity of geographic data are limited to the county level due to irregular entry of zip code data by prescribing pharmacies.

Conclusions: In the state of Texas over the studied time period, there was strong correlation for higher rates of opioid prescribing as white population increased despite overall decreased opioid prescribing starting in 2017. Until 2017, this correlation grew stronger as low-income white population increased.

Key words: Opioid, opioid epidemic, opioid utilization

Pain Physician 2019: 22:E425-E433 
T he current opioid epidemic confronting the United States is a substantial public health challenge. Annual deaths in the United States from opioids are estimated to increase to 72,000 this year (1), and $75 \%$ of opioid abusers report their first exposure to opioids was in the form of a prescription opioid (2). Although multiple factors led to the rise of this epidemic, it is without question associated with the rise in opioid prescribing.

Texas is the second largest state in the United States by both land area and population (3). It contains 3 of the top 10 largest cities in the United States (4), yet also contains 3 of the 4 least densely populated counties in the United States (5). It is also the seventh most ethnically diverse state (6). It has a substantially higher Hispanic population compared with the United States as a whole, but similar proportion of white and black residents $(7,8)$. Given such a large and diverse population, both ethnically and geographically, we hypothesized that a population-level assessment of opioid prescribing habits in Texas may yield valuable, actionable information for combating the opioid epidemic both for Texas and potentially the entire United States.

In this present study, using data extracted from both the Texas Prescription Drug Monitoring Programs (PDMP) and the US Census Bureau, we evaluated the trends in opioid prescribing in Texas and its interplay with population variables. The aim of this study was to evaluate geographic, ethnic, income, and time-related trends or disparities in opioid prescribing habits that would inform public health experts and policy makers of potential interventions they could pursue on a population level to more effectively address the opioid epidemic.

\section{Methods}

We obtained PDMP data related to controlled substance prescriptions from April 2015 to May 2018 from the Texas State Board of Pharmacy. We grouped individual drugs by mechanisms of action (Table 1). We removed the very small number of prescriptions that were missing drug and schedule information (coded as "unspecified"). We aggregated the prescription data by county in which the opioid prescription was filled per month. We obtained the data based on the county of the dispensing pharmacy rather than the zip code of the patient's home address because patient zip code data were often incorrectly entered at the pharmacy level or not entered at all. We assumed that, in most cases, the patient would fill the prescription in or near their residential county, although this necessarily limits the granularity of conclusions regarding geographic trends in prescribing habits.

We integrated the PDMP data with demographic data from the US Census Bureau to explore what population-level characteristics drive rates of opioid prescription. We used the US Census Bureau data (9) to calculate the monthly opioid prescription rates per capita and their association with economic and ethnic and racial characteristics within a county.

We used standard linear regression modeling to understand how the prescribing patterns are changing with geography and drug type. We predicted per capita opioid prescription rate with time for each individual drug schedule or county to find which of these are changing. When we fit many models to explore which drugs or counties are changing in prescription rate, we adjusted $\mathrm{P}$ values for multiple comparisons using the standard Bonferroni correction.

We used multiple linear regression to understand how prescription rates are associated with these factors. In this case, we fit a single model to all the counties to understand how their characteristics affect the opioid prescription rate. We explored including and excluding the various relevant predictors to build the best explanatory model that can account for the relationships that exist in this integrated PDMP and US Census Bureau dataset.

The modeling in this article is straightforward single and multiple linear regression. In all cases, the predicted quantity is the per capita prescription rate for controlled substances, a simple continuous variable, so we used standard linear modeling approaches in $R(R$ Foundation for Statistical Computing, Vienna, Austria) with standard statistical cutoffs for significance when applicable. The analysis for this article was performed entirely in $R$ using the packages dplyr, tidyr, broom, ggplot2, purrr, readxl, googlesheets, lubridate, sf, viridis, and huxtable. US Census Bureau data were accessed using the $\mathrm{R}$ package tidycensus.

\section{RESULTS}

The median number of controlled substance pills prescribed per month between April 2015 and May 2018 was 200 million. The number of pills changed at approximately $-0.00751 \%$ per month, or a $0.090 \%$ decrease per year. This change/decrease is lower than the rate of growth of the Texas population, estimated by the US Census Bureau at approximately $1.4 \%$ annually (10). 
Table 1 shows the proportion of total pills prescribed in the top 10 drug categories. We found that schedule IV drugs accounted for the most doses prescribed, with schedule II drugs close behind, and both are decreasing in prescribing prevalence $(P<0.005)$. The decrease is noticeable near the beginning of 2017 (Fig. 1). The most commonly prescribed drugs that exhibited significant change in prescribing volume are amphetamines (increasing) and barbiturates (decreasing).

Figure 2 demonstrates that there is significant geographic variation in the median monthly opioid pills prescribed per capita. For example, the opioid prescription rate in Tarrant County (the third most populous county) is almost $40 \%$ higher than the rate in Harris County (the most populous county). There are low rates in the far southern part of the state (i.e., the Rio Grande Valley) and high rates in northern and eastern counties. This geographic variation in opioid prescription rate has strong relationships with race and ethnicity. Most counties in Texas are either majority white or majority Hispanic, and the proportion of white residents within a county correlate with a higher median monthly opioid prescription rate.
We created multiple linear regression models to predict the median monthly per capita prescriptions with predictors including race, income, time, and total population to understand the influence of each of

Table 1. Top 10 drug categories for controlled prescriptions in Texas from April 2015 and May 2018.

\begin{tabular}{|l|c|}
\hline \multicolumn{1}{|c|}{ Drug } & $\begin{array}{c}\text { Total Pills over this } \\
\text { Time Period (\%) }\end{array}$ \\
\hline Opioid & $55.9 \%$ \\
\hline Benzodiazepine & $19.3 \%$ \\
\hline Amphetamine & $11.1 \%$ \\
\hline Sedative & $3.8 \%$ \\
\hline GABA receptor agonist & $3.8 \%$ \\
\hline Barbiturate & $2.1 \%$ \\
\hline Anabolic steroid & $1.3 \%$ \\
\hline Stimulant & $1.0 \%$ \\
\hline Anticonvulsant & $0.6 \%$ \\
\hline Nonbenzodiazepine hypnotic & $0.5 \%$ \\
\hline
\end{tabular}

Abbreviations: GABA, gamma aminobutyric acid

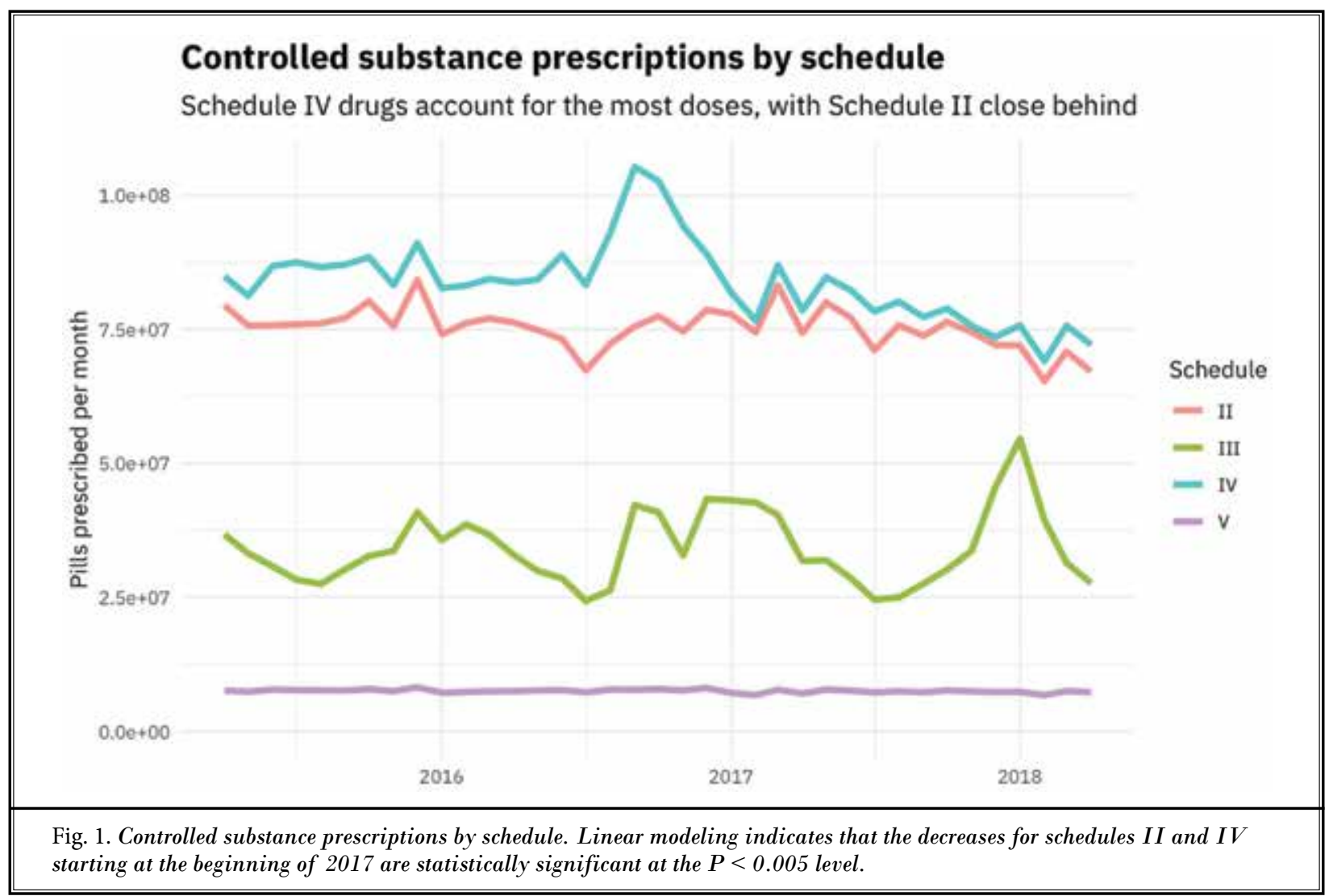




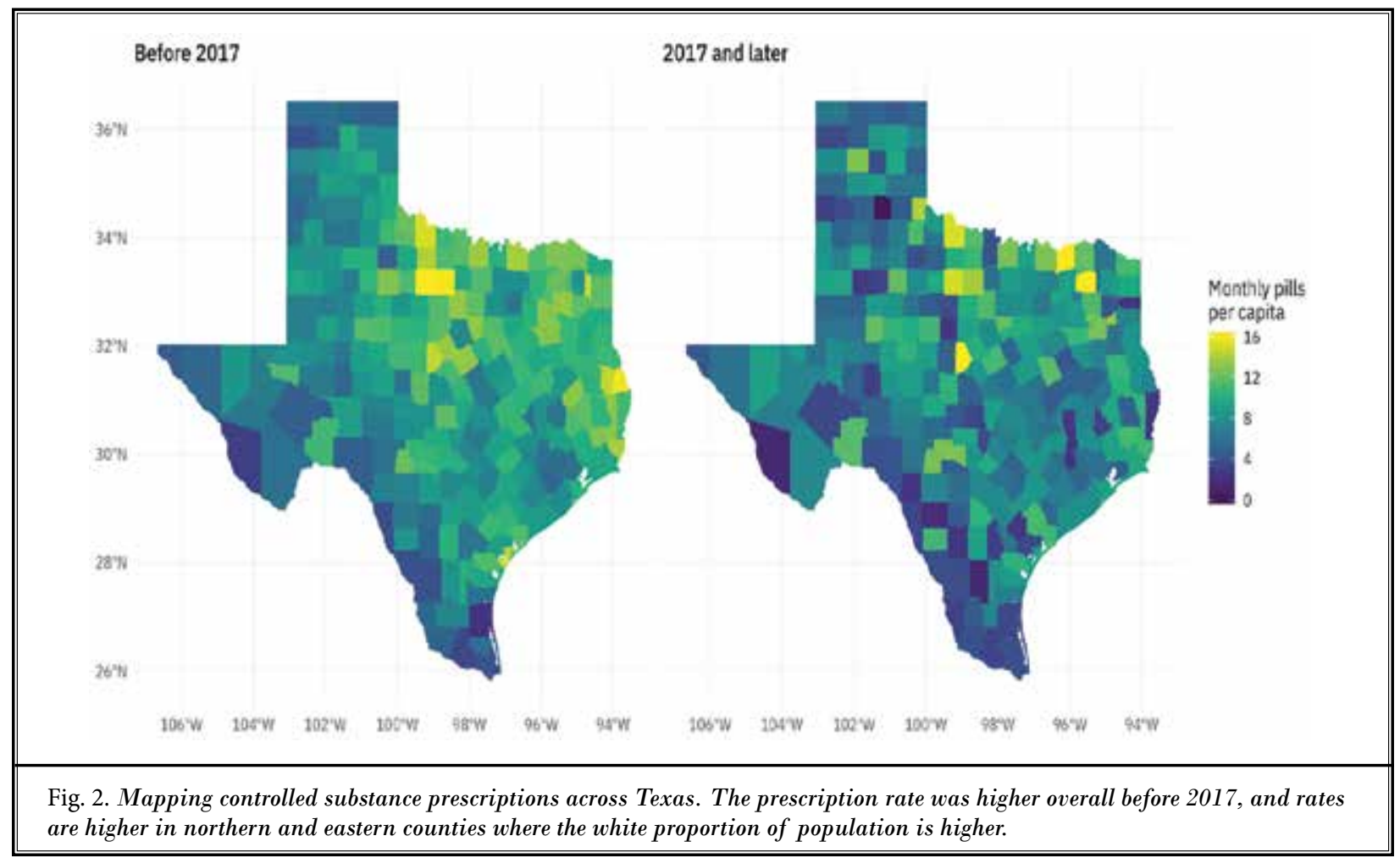

these factors. We found that there is no clear relationship between median household income by county (11) and prescriptions alone. Using this explanatory model, we found that opioid prescriptions were higher before 2017, in more populous counties (on a logarithmic scale), counties with predominantly white population, and poorer counties. When controlling for county population, the effects from low income and white population become stronger. When we do not account for ethnicity, there is no significant effect from income and the model metrics indicate a poor fit.

Model metrics such as adjusted R2 and log likelihood indicate that the model including income, percent white population, time, and total population on a log scale provides the most explanatory power for opioid prescriptions (Table 2). Using the proportion of population that is Hispanic gives a model that is similarly explanatory, but opposite in effect. That is, increasing percent Hispanic population within a county is associated with lower opioid prescribing rates. Effects with black and Asian ethnicity are not clear with this dataset. Overall, the R2 of these models is not extremely high (the best model has an adjusted R2 of 0.36 ) because these models are estimating population-level characteristics and there is significant county-to-county varia- tion that is not explained by these 4 predictors alone. However, the population-level trends are statistically significant and with the effect sizes at the levels shown here.

We can more directly explore the factors involved in this explanatory model (income, ethnicity, time) visually in Fig. 3. This plot illustrates the relationship between white population percentage and income, and how that has changed with time. The first effect to notice is that the greater the white population in a county the higher the rate of opioid prescriptions. This is true both before and after 2017, and for both lowincome and high-income groups. The second effect is to compare the slopes of the 2 lines. Before 2017, the slope was shallower for higher income counties (above the median in Texas), but the slope was steeper (i.e., the increase in prescription rate with white percentage was more dramatic) in the lower income counties (below the median in Texas). For 2017 and later, there is no longer a noticeable difference between low-income and high-income counties, although the trend with white population remains. Therefore at the county level in Texas, opioid prescriptions are associated mainly with an increasing proportion of white residents; before 2017, this correlation was stronger 
Population Analysis of Opioid Prescribing Habits

Table 2. Model 4 is the best fit to the data for predicting per capita prescription rate and includes income (scaled by $\$ 100,000$ ), the proportion of white population, time (2017 and later vs. before 2017), and the total population on a logarithmic scale.

\begin{tabular}{|c|c|c|c|c|}
\hline & (1) & (2) & (3) & (4) \\
\hline \multirow{2}{*}{ (Intercept) } & $5.674^{*}$ & $6.503^{*}$ & $8.017^{*}$ & $3.244^{*}$ \\
\hline & $(0.536)$ & $(0.518)$ & $(0.837)$ & $(0.769)$ \\
\hline \multirow{2}{*}{ Income (per $\$ 100 \mathrm{~K}$ ) } & $-3.285^{\star *}$ & $-3.302^{*}$ & -0.439 & $-5.046^{\star}$ \\
\hline & $(1.040)$ & $(0.984)$ & $(1.145)$ & $(1.005)$ \\
\hline \multirow{2}{*}{ White \% } & $7.053^{*}$ & $7.066^{*}$ & & $7.786^{*}$ \\
\hline & $(0.554)$ & $(0.524)$ & & $(0.525)$ \\
\hline \multirow{2}{*}{2017 and later } & & $-1.655^{\star}$ & $-1.644^{*}$ & $-1.653^{*}$ \\
\hline & & $(0.213)$ & $(0.248)$ & $(0.207)$ \\
\hline \multirow{2}{*}{$\log$ (TotalPop) } & & & 0.131 & $0.372^{*}$ \\
\hline & & & $(0.077)$ & $(0.066)$ \\
\hline $\mathrm{N}$ & 507 & 507 & 507 & 507 \\
\hline $\mathrm{R} 2$ & 0.243 & 0.324 & 0.085 & 0.364 \\
\hline $\log L i k$ & -1190.314 & -1161.676 & -1238.385 & -1146.313 \\
\hline AIC & 2388.629 & 2333.352 & 2486.769 & 2304.626 \\
\hline
\end{tabular}

Abbreviation: AIC, XXXX. \{AU: Please provide spell out of AIC $\}$

${ }^{\star} \mathrm{P}<0.001$.

${ }^{* *} \mathrm{P}<0.01$

${ }^{* * *} \mathrm{P}<0.05$. $\left\{\right.$ AU: Please note that symbols ${ }^{*}$ and ${ }^{* * *}$ were switched, but there is no indication for ${ }^{* * *}$ in the Table

\section{White population, income, and controlled substance usage}

Before 2017, the more white a county was, the more low income was associated with more controlled substance usage

$$
\text { - Heghincome }- \text { tow incorse }
$$

Before 2017

2017 and later

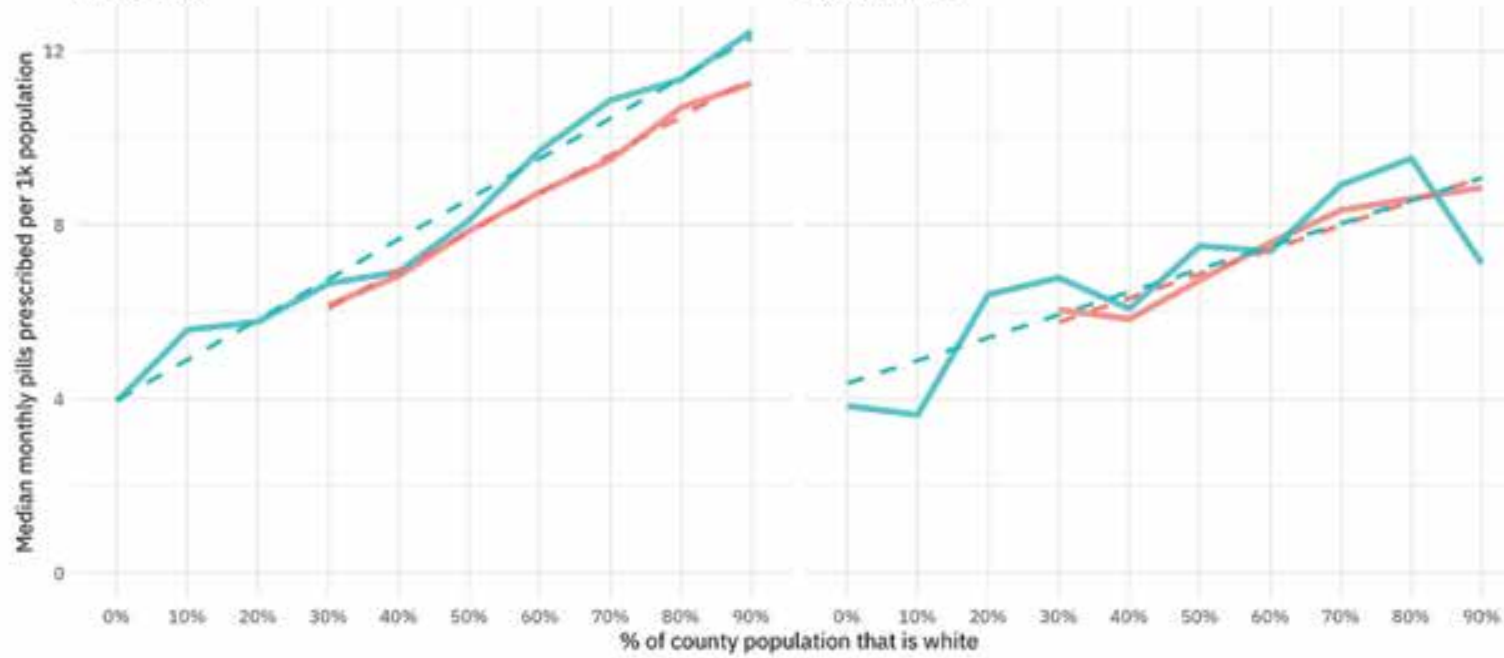

Fig. 3. How population, income, and controlled substance usage are related. The more predominant a white population is in a county, the higher the rate of controlled substance prescriptions there. 
when the typical income of a population was below the median.

\section{Discussion}

Our study revealed a strong positive correlation in opioid prescription rates in counties with higher white population density. Alternatively, there was a strong negative correlation in opioid prescription rates in counties with higher Hispanic population density. Our analysis clearly demonstrates that there is an ethnic disparity or difference in opioid prescription rates, although ethnicity as the causative factor for this difference per se cannot be addressed directly. The relationship between income levels and opioid prescription rates did not hold when ethnicity was not considered. Of note, no counties in Texas have a majority black or majority Asian population, so our evaluation cannot provide insight for these racial and ethnic populations.

Although disparity has been shown to exist throughout the health care spectrum, the treatment of pain has unique challenges that may contribute to these disparities. Pain is, by definition, subjective in both its experience and, to a large degree, in evaluation. Ubiquitous use of self-reported numeric pain scales to give a single, objective-appearing metric on what is, at its core, a subjective biopsychosocial experience has resulted in a lack of trust of such scales by health care providers (12). Thus self-report of increasing levels of pain, especially in the setting of limited objective evidence to support such self-report, often leads health care providers to fall back on heuristic or stereotype-based decision processes (13). This introduces the potential for implicit bias, one's unconscious attitudes and beliefs toward others, to shape the decision process and has been implicated as one influence on racial and ethnic disparities in analgesic prescribing (14). Other investigators have shown that educational efforts that draw attention to the presence of these often unnamed, and therefore invisible, biases can help to ameliorate their deleterious effects (15-17). Additionally, a study assessing the influence of ethnicity on prescribing habits in the emergency department (ED), albeit using responses to clinical vignettes and not actual prescribing data, found that the observed difference between ethnic groups may have less to do with ethnicity per se, but based on the presence or absence of desirable social traits, such as indicators of higher socioeconomic status, role demands, and a relationship with a primary care provider (18). A retrospective study found that although both black and white patients were given analgesics of some sort for various painful conditions in an ED setting, white patients were more likely to be prescribed an opioid for low back pain, although this disparity was not observed for long bone fractures or migraines (14).

One other potential source of the observed disparity in opioid prescribing between white and Hispanic populations is language. The lowest observed per capita opioid prescribing rates were in counties with the highest Hispanic populations and in the Texas counties along the US-Mexico border. Although beyond the scope of our analysis, it is reasonable to suppose that a language barrier between patient and provider was more common in this population and geographic area. Other studies have found that language barriers are a common source of disparity in health care access $(19,20)$. Finally, factors such as sociological or cultural differences in pain perception and communication behaviors with health care providers could impact the observed disparities (21).

The relationship between the apparent disparity in analgesic prescribing and how minority communities have been impacted by the opioid epidemic is complex. Several studies using alternative data sources have corroborated our findings of lower opioid prescribing rates in non-white populations (21-26), although one study did find higher opioid prescribing in non-white and rural populations (27). One study showed higher rates of disorders related to opioid abuse and dependence (e.g., alcohol abuse, benzodiazepine use, affective disorders, sleep disorders) in white populations compared with non-white populations, yet simultaneously higher rates of opioid adverse effects and accidental poisonings in non-white populations (21). Also, hospitalizations for opioid and heroin poisoning were shown to be more likely in lower-income white populations (28). Lower income may also be a risk factor for opioid intoxication (21) and poisoning (29). Additionally, low-income rural populations showed that a co-diagnosis of depression more than tripled the likelihood that a patient would also have a prescription for opioids (30). Whether this effect was owing to elevated depression in chronic nonmalignant pain or other factors, such as "chemical coping," were not addressed. It is unclear whether such a relationship exists in higher income or urban and suburban populations but warrants further study as potential contributing factors. Furthermore, opioid-related mortality from the mid-1990s to 2010 was largely driven by growth in prescription opioid use among white populations (31). In recent years, although the 
growth in opioid abuse has mostly been due to significant growth in heroin abuse, $90 \%$ of new heroin users over the past decade have been white and tended to switch from prescription opioid use to heroin owing to increased availability and lower cost (32). Also, since 2010, there has been increased opioid-related mortality specifically attributable to heroin and synthetic opioid (e.g., fentanyl) use that is correlated with prior use of prescription opioids (33). Therefore although the racial and ethnic disparity in opioid prescribing may have been detrimental to the treatment of pain in non-white populations, it also appears to have had an unintended sparing effect on these populations in regard to the relative impact of both prescription opioid and heroin abuse and resulting morbidity and mortality.

The decrease in opioid prescriptions in 2017 noted in our study has also been noted in nationwide analysis of opioid prescriptions, which noted an overall decrease of $8.9 \%$ from 2016 to 2017, and a decrease of $22.2 \%$ from 2013 to 2017 (34). In the current study, opioids tracked in the Texas PDMP decreased overall by $4.7 \%$ from 2016 to 2017, whereas schedule IV drugs decreased the most and accounted for most of the drop in Texas, decreasing by over $11 \%$. The reason for such a rapid drop in opioid prescriptions is unclear, but it is likely multifactorial. The possible factors include the growing national awareness of the severity of opioidrelated overdoses (35), increase in grant programs (36), increase use of PDMPs by health care providers (37), and efforts by licensing agencies (e.g., Texas Medical Board) (36), and state legislators (38).

This study has some limitations. We could not obtain geographic granularity beyond the county level (e.g., zip codes of recipients) because of inadequate documentation at the pharmacy level. Although still yielding valuable data, this does place a firm lower limit on the geographic analytic capabilities of our evaluation, an effect that would be most noticeable in higher density population centers. Also, the PDMP database does not include nonprescribed controlled substances or illicit drugs with similar mechanisms of action (e.g., heroin), so our analysis gives an incomplete picture of the full spectrum of illicit drugs, opioids, or other controlled substance use in the population because we cannot account for diversion after a prescription is given to a patient, diversion from outside Texas, or illicit drug use. Furthermore, a small subset of pharmacies are exempt from reporting to the Texas PDMP, such as Veterans' Affairs pharmacies and methadone clinics.
We also would like to have been able to analyze data on prescribing habits based on physician specialty, but this specific variable is not captured by the Texas PDMP. Finally, we assessed PDMP data from Texas, but not from other states. Each state maintains a separate PDMP, and access is often limited to licensed providers in that state. These barriers therefore limit access to broader PDMP data, so larger studies involving multiple states are difficult to pursue. It is also difficult to gain access to data over longer time frames depending on state laws mandating maintenance of PDMP data. In Texas, for instance, the PDMP data must be maintained for 3 years according to legislative mandate, but beyond that time frame it may be lost, as funding does not cover database storage and maintenance. This perhaps unintended consequence of the legislative mandate limits the ability of public health experts and policy makers to gain a wider perspective of the role of prescribed controlled substances in the larger opioid epidemic over broader time frames, and thus limits the ability to assess the long-term effects of policy changes.

\section{Conclusions}

In conclusion, analysis of the Texas PDMP showed that there is a strong geographic trend in controlled substance prescribing across the state of Texas. This trend is driven by county-level ethnic variations, namely that as the proportion of white residents increases in a county so does the rate of opioid prescribing. In addition, although income does not correlate with prescribing rates at a population level, prescribing rates increase faster as median-income white population density compared with greater high-income white population density; this result was most clear for pre-2017 data. Public health experts and policy makers should take note of these trends when crafting interventions to combat the opioid epidemic.

Future research should focus on confirming the observed relationship in other populations over greater time periods. We suggest that PDMP data be maintained in perpetuity so that long-term analysis is possible to assess the efficacy of public health interventions over longer time frames. In addition, a national PDMP, or at least interoperability between states' PDMPs, would allow nationwide population-level trends to be evaluated, and would especially benefit physicians practicing along state borders to more effectively assess for aberrant controlled substance procurement behaviors in bordering states. 


\section{References}

1. Ahmad FB, Rossen LM, Spencer MR, Warner M, Sutton P. Provisional drug overdose death counts. National Center for Health Statistics. 2018.

2. Compton WM, Jones CM, Baldwin GT. Relationship between nonmedical prescription-opioid use and heroin use. $N$ Engl J Med 2016; 374:154-163.

3. U.S. Census Bureau. Program surveys and population tables 2010-2017 www2.census.gov/programs-surveys/ popest/tables/2010-2017/state/totals/ nst-est2017-01.xlsx. Accessed August 26, 2019.

4. U.S. Census Bureau. Annual Estimates of the Resident Population: April 1, 2010 to July 1, $2017 \mathrm{https://www.census.gov/}$ newsroom/press-releases/2018/estimates-cities.html\#table3. Accessed August, 26, 2019.

5. U.S. Census Bureau. Big and Small America. https://www.census.gov/library/stories/2017/10/big-and-smallcounties.html. Accessed August, 26, 2019.

6. Lee BA, Martin MJR, Mathews SA, Farrell CR. State-level changes in US racial and ethnic diversity, 1980-2015: A universal trend? Demogr Res 2017; 37:1031-1048.

7. U.S. Census Bureau. Quick Facts - Texas. www.census.gov/quickfacts/TX. Accessed August 26, 2019.

8. U.S. Census Bureau. Quick Facts. www. census.gov/quickfacts/fact/table/US/ PSTo45216. Accessed August 26, 2019.

9. United States Census Bureau."Bo1003: Total Population." 2011-2015 American Community Survey. U.S. Census Bureau's American Community Survey Office, 2018.

10. U.S. Census Bureau. Idaho is Nation's Fastest-Growing State, Census Bureau Reports. www.census.gov/newsroom/ press-releases/2017/estimates-idaho. html. Accessed August 26, 2019.

11. United States Census Bureau. "B19013: Median Household Income." 2011-2015 American Community Survey. U.S. Census Bureau's American Community Survey Office, 2018.

12. Chibnall JT, Tait RC, Ross LR. The effects of medical evidence and pain intensity on medical student judgments of chronic pain patients. J Behav Med 1997; 20:257-271.

13. Tait RC, Chibnall JT. Racial/ethnic disparities in the assessment and treat- ment of pain: Psychosocial perspectives. Am Psychol 2014; 69:131-141.

14. Dickason RM, Chauhan V, Mor A, Ibler E, Kuehnle S, Mahoney D, Armbrecht E, Dalawari P. Racial differences in opiate administration for pain relief at an academic emergency department. West J Emerg Med 2015; 16:372-380.

15. Ewen SC, Barrett J, Paul D, Askew D, Webb G, Wilkin A. When a patient's ethnicity is declared, medical students decision-making processes are affected. Intern Med J 2015; 45:805-812.

16. Nelson SC, Prasad S, Hackman HW. Training providers on issues of race and racism improve health care equity. Pediatr Blood Cancer 2015; 62:915-917.

17. White-Davis T, Edgoose J, Brown Speights JS, Fraser K, Ring JM, Guh J, Saba GW. Addressing racism in medical education an interactive training module. Fam Med 2018; 50:364-368.

18. Tamayo-Sarver JH, Dawson NV, Hinze SW, Cydulka RK, Wilgton RS, Albert JM, Ibrahim SA, Baker DW. The effect of race/ ethnicity and desirable social characteristics on physicians' decisions to prescribe opioid analgesics. Acad Emerg Med 2003; 10:1239-1248.

19. Bradshaw M, Tomany-Korman S, Flores G. Language barriers to prescriptions for patients with limited English proficiency: A survey of pharmacies. Pediatrics 2007; 120:e225-e235.

20. Ponce NA, Hays RD, Cunningham WE. Linguistic disparities in health care access and health status among older adults. J Gen Intern Med 2006; 21:786-791.

21. Feng J, Iser JP, Yang W. Medical encounters for opioid-related intoxications in southern Nevada: Sociodemographic and clinical correlates. BMC Health Serv Res 2016; 16:438.

22. Chen I, Kurz J, Pasanen M, Gaselis C Panda M, Staton LJ, O'Rorke J, Menon M, Genao I, Wood J, Mechaber AJ, Rosenberg E, Carey T, Calleson D, Cykert S. Racial differences in opioid use for chronic nonmalignant pain. J Gen Intern Med 2005; 20:593-598.

23. Burgess DJ, Nelson DB, Gravely AA, Bair MJ, Kerns RD, Higgins DM, van Ryn M, Farmer M, Partin M. Racial differences in prescription of opioid analgesics for chronic noncancer pain in a national sample of veterans. J Pain 2014; 15:447-455.
24. Pletcher MJ, Kertesz SG, Kohn MA, Gonzalez R. Trends in opioid prescribing by race/ethnicity for patients seeking care in US emergency departments. JAMA 2008; 299:70-78.

25. Hausmann LR, Gao S, Lee ES, Kwoh CK. Racial disparities in the monitoring of patients on chronic opioid therapy. Pain 2013; 154:46-52.

26. Shavers VL, Bakos A, Sheppard VB. Race, ethnicity, and pain among the U.S. adult population. J Health Care Poor Underserved 2010; 21:177-220.

27. Prunuske JP, St. Hill CA, Hager KD, Lemieux AM, Swanoski MT, Anderson GW, Lutifiyya MN. Opioid prescribing patterns for non-malignant chronic pain for rural versus non-rural US adults: A population-based study using 2010 NAMCS data. BMC Health Serv Res 2014; 14:563.

28. Song Z. Mortality quadrupled among opioid-driven hospitalizations, notably within lower-income and disable white populations. Health Aff (Millwood) 2017; 36:2054-2061.

29. Cerda M, Gaidus A, Keyes KM, Ponicki W, Martins S, Galea S, Gruenewald P. Prescription opioid poisoning across urban and rural areas: Identifying vulnerable groups and geographic areas. Addiction 2017; 112:103-112.

30. Kapoor S, Thorne BE. Healthcare use and prescription of opioids in rural residents with pain. Rural Remote Health 2014; 14:2879.

31. Alexander MJ, Kiang MV, Barbieri M. Trends in black and white opioid mortality in the United States, 1979-2015. Epidemiology 2018; 28:707-715.

32. Cicero TJ, Ellis MS, Surratt HL, Kurtz SP. The changing face of heroin use in the United States: A retrospective analysis of the past 50 years. JAMA Psychiatry 2014; 71:821-826.

33. Jones CM, Logan J, Gladden RM, Bohm MK. Vital signs: Demographic and substance use trends among heroin usersUnited States, 2002-2013. MMWR Morb Mortal Wkly Rep 2015; 64:719-725.

34. IQVIA. National and State-level Opioid Prescribing Analysis. www.texmed.org// uploadedFiles/Current/2016_Public_ Health/Opioids/IQVIA\%20Opioid\%20 state $\% 20$ and $\% 20$ payer\%2oviews $\% 20$ 2017\%20FINAL.PDF. Accessed August 26, 2019. 
35. White House Briefing Statement. President Donald J. Trump is Taking Action on Drug Addiction and the Opioid Crisis. Octo 26, 2017. www.whitehouse. gov/briefings-statements/presidentdonald-j-trump-taking-action-drug-addiction-opioid-crisis/. Accessed August 26, 2019.

36. Substance Abuse and Mental Health
Services Administration. SAMHSA to award three new grants to address the opioid epidemic. https://www.samhsa.gov/newsroom/press-announcements/201711290500 Accessed August, 26, 2019.

37. American Medical Association. Opioid Task Force 2019 Progress Report. https://www.ama-assn.org/system/ files/2019-06/opioid-task-force-progress-report.pdf. Accessed August 26, 2019.

38. Texas Medical Board. State and National Focus on Combatting the Current Opioid Epidemic. www.tmb.state.tx.us/ dl/209310BC-7307-EF80-6049-3FDC28CF182D. Accessed August 26, 2019. 
